Pacific Journal of Mathematics

A UNIQUENESS THEOREM FOR SECOND ORDE 


\title{
A UNIQUENESS THEOREM FOR SECOND ORDER QUASILINEAR HYPERBOLIC EQUATIONS
}

\author{
A. E. HURD
}

A uniqueness theorem is proved for weak solutions of quasilinear second-order hyperbolic equations of the form

$$
u_{t t}-\sum_{i=1}^{n} \frac{\partial}{\partial x_{i}} a^{i}\left(x, t, u, u_{1} \cdots, u_{n}\right)=b(x, t, u)
$$

in many space variables. The weak solutions are assumed to satisfy a time-wise upper Lipschitz bound

$$
\frac{u_{k}\left(x, t_{1}\right)-u_{k}\left(x, t_{2}\right)}{t_{1}-t_{2}} \leqq K(t)
$$

for all $0<t \leqq t_{1}, t_{2}$ where $K(t)$ is an $L^{1}$-function. Together with the obvious assumptions, the equation is supposed to satisfy a symmetry condition

$$
\frac{\partial a^{i}}{\partial u_{j}}=\frac{\partial a^{j}}{\partial u_{i}}
$$

along with convexity of the $a^{i}$ in $u$ and $u_{k}$. As a corollary, a uniqueness theorem for systems proved by Oleinik is generalized.

In recent years a number of authors have studied quasilinear hyperbolic equations and systems with the goal of obtaining general existence and uniqueness theorems for the initial problem. Regular or smooth solutions do not usually exist for these problems, and so one tries to establish the existence of weak or generalized solutions of various types. The uniqueness question for such solutions is then somewhat more tenuous than that for smooth solutions, and usually involves the assumption of some sort of one-sided Lipschitz estimate on the solution.

The first comprehensive attack on these problems began with Oleinnik's paper [6] in which she established existence and uniqueness results for generalized solutions of first order equations of the form

$$
\frac{\partial u}{\partial t}+\frac{\partial \varphi}{\partial x}(x, t, u)+\psi(x, t, u)=0 \text {. }
$$

The function $\varphi$ was subject to a convexity assumption

$$
\frac{\partial^{2} \varphi}{\partial u^{2}} \geqq 0 \text {. }
$$

To prove uniqueness she used a variant of the method of Holmgren 
(see [3]), assuming that the generalized solutions $u$ were bounded measurable, and satisfied a Lipschitz bound of the form

$$
\frac{u\left(x_{1}, t\right)-u\left(x_{2}, t\right)}{x_{1}-x_{2}} \leqq K\left(x_{1}, x_{2}, t\right) \text {. }
$$

Since Oleǐnik's paper much effort has been directed to generalizing her results in two directions;

(a) to systems of first order equations (see e.g. [4]), and

(b) to equations in many space variables ([1], [2]).

However, little progress has been made on a corresponding general theory for higher order equations, with no existence theorems having yet been produced.

This paper is devoted to proving a uniqueness theorem for weak solutions of the initial value problem for second order symmetric (Assumption II) quasilinear hyperbolic equations in several space variables. A variant of the Holmgren method is again used, except that energy estimates are used in place of pointwise estimates. The same method has been applied to symmetric first order systems in [5]. We also require convexity-type assumptions on the equation (Assumption IV). But in interesting contrast with the case of first order equations, we are led to impose time-wise Lipschitz bounds on the solution (Assumption B) in place of the space-wise Lipschitz bounds (3).

In the last part of the paper our result is used to generalize a uniqueness theorem for a hyperbolic system of two first order quasilinear equations which was obtained by Oleinik [7]. The generalization essentially amounts to weakening the convexity condition, and replacing a constant Lipschitz bound by an $L^{1}$ function. It would seem that more substantial extensions of Oleinik's result are possible using the technique presented here.

2. The uniqueness theorem. In the region

$$
D=\left\{(x, t): x=\left(x_{1}, \cdots, x_{n}\right) \in R_{n} ; t \text { real, } 0<t<\infty\right\}
$$

we consider the second order quasilinear hyperbolic equation

$$
u_{t t}-\sum_{i=1}^{n} \frac{\partial}{\partial x_{i}} a^{i}\left(x, t, u, u_{1}, \cdots, u_{n}\right)-b(x, t, u)=0
$$

for the function $u(x, t)$, where we have used the notation

$$
\frac{\partial \psi}{\partial x_{k}}=\psi_{k} \text { and } \frac{\partial \psi}{\partial t}=\psi_{t} .
$$

The equation will be subject to the following assumptions.

I. The functions $a^{i}\left(x, t, u, u_{k}\right)$ and $b(x, t, u)$ are defined for all 
$x, t, u, u_{k}$ satisfying $-\infty<x, u, u_{k}<\infty, 0<t<\infty$, and are differentiable with respect to these variables, the derivatives being uniformly Lipschitz continuous on compact subsets of $\bar{D}$. Also $\partial a^{i} / \partial u_{j}$ is continuously differentiable with respect to $t, u$ and $u_{k}$.

II. (Symmetry) If

$$
\frac{\partial a^{i}}{\partial u_{j}}\left(x, t, u, u_{k}\right)=a^{i j}\left(x, t, u, u_{k}\right)
$$

then

$$
a^{i j}\left(x, t, u, u_{k}\right)=a^{j i}\left(x, t, u, u_{k}\right) .
$$

III. Given positive constants $M$ and $T$ there are corresponding constants $c_{1}>0$ and $c_{2}>0$ such that

$$
\begin{aligned}
c_{1} \sum_{i=1}^{n} \xi_{i}^{2} & \geqq \sum_{i, j=1}^{n} a^{i j}\left(x, t, u, u_{k}\right) \xi_{i} \xi_{j} \\
& \geqq c_{2} \sum_{i=1}^{n} \xi_{i}^{2}
\end{aligned}
$$

for all vectors $\left(\xi_{1}, \cdots, \xi_{n}\right)$ if $(x, t) \in R_{n} \times[0, T]$ and

$$
|u|+\sum_{j=1}^{n}\left|u_{j}\right| \leqq M
$$

IV. (Convexity). For all vectors $\left(\xi_{1}, \cdots, \xi_{n}\right)$ we have

$$
\sum_{i, j=1}^{n} \frac{\partial \alpha^{i j}}{\partial u}\left(x, t, u, u_{k}\right) \xi_{i} \xi_{j} \leqq 0
$$

and

$$
\sum_{i, j=1}^{n} \frac{\partial a^{i j}}{\partial u_{k}}\left(x, t, u, u_{k}\right) \xi_{i} \xi_{j} \leqq 0 \quad(k=1, \cdots, n) .
$$

V. The functions

$$
\frac{\partial a^{i j}}{\partial t}\left(x, t, u, u_{k}\right), \frac{\partial a^{i}}{\partial u}\left(x, t, u, u_{k}\right) \text { and } \frac{\partial b}{\partial u}(x, t, u)
$$

are uniformly bounded on compact subsets of $\left(x, t, u, u_{k}\right)$ space.

We will be concerned with weak solutions of (4).

DEFINITION. Let $f(x)$ and $g(x)$ be essentially bounded measurable functions on $R_{n}$. A weak solution $u(x, t)$ of (4) on $D$ with initial conditions

$$
u(x, 0)=f(x) \text { and } \frac{\partial u}{\partial t}(x, 0)=g(x)
$$


is an essentially bounded measurable function possessing essentially bounded measurable weak (i.e., distribution) derivatives $u_{k}$ and $u_{t}$ a.e. on $D$ which satisfy the following conditions:

A. For every twice continuously differentiable test function $\varphi(x, t)$ on $\bar{D}$ which vanishes for large $|x|+\mathrm{t},\left(|x|=\left(x_{1}^{2}+\cdots+x_{n}^{2}\right)^{1 / 2}\right)$ we have

$$
\begin{aligned}
\iint_{D}\left[u \varphi_{t t}\right. & \left.+\sum_{i=1}^{n} a^{i}\left(x, t, u, u_{k}\right) \varphi_{i}-b(x, t, u,) \varphi\right] d x d t \\
& +\int_{R_{n}} f(x) \varphi_{t}(x, 0) d x-\int_{R_{n}} g(x) \varphi(x, 0) d x=0 .
\end{aligned}
$$

B. Given any compact subset $R$ of $R_{n}$, and $T, 0 \leqq T<\infty$ there is a function $K(t) \in L^{1}(0, T)$ such that

$$
\frac{u_{k}\left(x, t_{1}\right)-u_{k}\left(x, t_{2}\right)}{t_{1}-t_{2}} \leqq K(t) \quad(k=1, \cdots, n)
$$

holds a.e. for all $x \in R$, and $0<t_{1}<t_{2} \leqq t<T .^{1}$

Any twice continuously differentiable (smooth) solution of (4) is a weak solution as is easily seen by applying the divergence theorem.

Before presenting the main result of the paper we establish a lemma concerning energy inequalities for twice continuously differentiable solutions of hyperbolic equations of the form

$$
\begin{aligned}
L \varphi= & \varphi_{t t}-\sum_{i, j=1}^{n}\left(\alpha^{i j}(x, t) \varphi_{i}\right)_{j} \\
& +\sum_{i=1}^{n} \alpha^{i}(x, t) \varphi_{i}-\beta(x, t) \varphi=F(x, t) .
\end{aligned}
$$

The energy of the solution $\varphi(x, t)$ at time $t$ is defined to be

$$
E(t)=\int_{R_{n}}\left[\phi_{t}^{2}(x, t)+\sum_{i=1}^{n} \phi_{i}^{2}(x, t)\right] d x .
$$

We will assume that our solution has uniformly bounded special support on any given finite time interval, in the sense that, given $T \geqq 0$ there is a rectangle $R \subset R_{n}$ such that the support of $\varphi(x, t)$ as a function of $x$ lies in $R$ for all $t, 0 \leqq t \leqq T$. A uniform bound on $E(t)$ for all such $t$ will be obtained under the following assumptions:

$I^{\prime}$. The functions $\alpha^{i j}(x, t), \alpha^{i}(x, t)$ and $\beta(x, t)$ are continuously differentiable functions of $x$ and $t$.

${ }^{1}$ Since $u$ has essentially bounded weak derivatives it follows (c.f. Serrin [9]) that

$$
\frac{u\left(x, t_{1}\right)-u\left(x, t_{2}\right)}{t_{1}-t_{2}} \leqq K
$$

holds a.e. in $R \times[0, T]$, where $K$ is some constant. 
II'. For all $i, j=1, \cdots, n$ we have $\alpha^{i j}(x, t)=\alpha^{j i}(x, t)$.

III'. There are constants $c_{1}>0$ and $c_{2}>0$ such that

$$
c_{1} \sum_{i=1}^{n} \xi_{i}^{2} \geqq \sum_{i, j=1}^{n} \alpha^{i j}(x, t) \xi_{i} \xi_{j} \geqq c_{2} \sum_{i=1}^{n} \xi_{i}^{2}
$$

for all $(x, t) \in R \times[0, T]$ and all vectors $\left(\xi_{1}, \cdots, \xi_{n}\right)$.

IV'. There is a function $K_{1}(t) \in L^{1}(0, T)$ such that

$$
\sum_{i, j=1}^{n} \alpha_{t}^{i j}(x, t) \xi_{i} \xi_{j} \leqq K_{1}(t) \sum_{i=1}^{n} \xi_{i}^{2}
$$

for all $(x, t) \in R \times[0, T]$ and all vectors $\left(\xi_{1}, \cdots, \xi_{n}\right)$.

$\mathrm{V}^{\prime}$. There are constants $A \geqq 0$ and $B \geqq 0$ such that

$$
\left|\alpha^{i}(x, t)\right| \leqq A, \quad|\beta(x, t)| \leqq B
$$

for all $(x, t) \in R \times[0, T]$.

VI'. The function $F(x, t)$ is square integrable on $R \times[0, T]$. Then we have

LEMmA. Under assumptions $\mathrm{I}^{\prime}$ through $\mathrm{VI}^{\prime}$ there is a constant $C$ such that

$$
E(\tau) \leqq C
$$

for all $\tau, 0 \leqq \tau \leqq T$.

Proof. We have

$$
\begin{aligned}
2 \varphi_{t} L \varphi= & {\left[\varphi_{t}^{2}+\sum_{i, j=1}^{n} \alpha^{i j} \varphi_{i} \varphi_{j}\right]_{t} } \\
& -2 \sum_{i, j=1}^{n}\left(\alpha^{i j} \varphi_{i} \varphi_{t}\right)_{j}-Q(x, t)
\end{aligned}
$$

where

$$
Q(x, t)=\sum_{i, j=1}^{n} \alpha_{t}^{i j} \varphi_{i} \varphi_{j}-2 \sum_{i=1}^{n} \alpha^{i} \varphi_{i} \varphi_{t}+2 \beta \varphi \varphi_{t} .
$$

Integrating (15) over $R \times[0, \tau], 0 \leqq \tau \leqq T$, using the divergence theorem, and the fact that $\varphi$ vanishes on the boundary of $R$ for all $t, 0 \leqq t \leqq \tau$, we obtain

$$
\begin{aligned}
\int_{R}\left[\varphi_{t}^{2}+\sum_{i, j=1}^{n} \alpha^{i j} \varphi_{i} \varphi_{j}\right]_{0}^{\tau} d x= & \int_{0}^{\tau} \int_{R} Q(x, t) d x d t \\
& +2 \int_{0}^{\tau} \int_{R} \varphi_{t} F(x, t) d x d t .
\end{aligned}
$$

Denoting all constants generically by $C$, there results 


$$
E(\tau) \leqq C\left[E(0)+\int_{0}^{\tau} \int_{R} Q(x, t) d x d t+2 \int_{0}^{\tau} \int_{R} \varphi_{t} F(x, t) d x d t\right] .
$$

Now to bound the right-hand side we note that from (13)

$$
\int_{0}^{\tau} \int_{R}\left[\sum_{i, j=1}^{n} \alpha_{t}^{i j} \varphi_{i} \varphi_{j}\right] d x d t \leqq \int_{0}^{\tau} K_{1}(t) E(t) d t
$$

and from (14)

$$
-\int_{0}^{\tau} \int_{R} 2\left[\sum_{i=1}^{n} \alpha^{i} \varphi_{i} \varphi_{t}\right] d x d t \leqq C \int_{0}^{\tau} E(t) d t
$$

Also from (14),

$$
\begin{aligned}
\int_{0}^{\tau} \int_{R} 2 \beta \varphi \varphi_{t} d x d t & \leqq C \int_{0}^{\tau} \int_{R}\left[\varphi^{2}+\varphi_{t}^{2}\right] d x d t \\
& \leqq C \int_{0}^{\tau} \int_{R_{n}} \varphi^{2} d x d t+C \int_{0}^{\tau} E(t) d t
\end{aligned}
$$

so that

$$
\begin{aligned}
& \int_{0}^{\tau} \int_{R} Q(x, t) d x d t \\
\leqq & \int_{0}^{t}[K(t)+C] E(t) d t+C \int_{0}^{\tau} \int_{R_{n}} \varphi^{2} d x d t .
\end{aligned}
$$

To estimate the last integral on the right we have

$$
\varphi(x, t)=\int_{0}^{t} \varphi_{s}(x, s) d s+\varphi(x, 0), \quad x \in R_{n} .
$$

By Schwarz' inequality

$$
\varphi^{2}(x, t) \leqq 2\left\{t \int_{0}^{\tau} \varphi_{s}^{2}(x, s) d s+\varphi^{2}(x, 0)\right\}
$$

and so

$$
\int_{0}^{\tau} \varphi^{2}(x, t) d t \leqq \int_{0}^{\tau}\left(\tau^{2}-t^{2}\right) \varphi_{t}^{2} d t+\tau \varphi^{2}(x, 0) .
$$

Thus

$$
\begin{aligned}
\int_{0}^{\tau} \int_{R} \varphi^{2}(x, t) d x d t & \leqq \int_{0}^{\tau}\left(\tau^{2}-t^{2}\right) E(t) \\
& +\tau \int_{R_{n}} \varphi^{2}(x, 0) d x
\end{aligned}
$$

yielding

$$
\begin{aligned}
\int_{0}^{\tau} \int_{R} Q(x, t) d x d t \leqq & \int_{0}^{\tau}\left[K(t)+\tau^{2}-t^{2}+C\right] E(t) d t \\
& +\tau \int_{R_{n}} \varphi^{2}(x, 0) d x .
\end{aligned}
$$


Finally,

$$
\begin{aligned}
2 \int_{0}^{\tau} \int_{R} \varphi_{t} F(x, t) d x d t \leqq & \int_{0}^{\tau} E(t) d t \\
& +\int_{0}^{\tau} \int_{R_{n}} F^{2}(x, t) d x d t,
\end{aligned}
$$

and so in all

$$
E(\tau) \leqq f(\tau)+\int_{0}^{\tau} \chi(t) E(t) d t
$$

where

$$
f(\tau)=C\left[E(0)+\tau \int_{R_{n}} \varphi^{2}(x, 0) d x+\int_{0}^{\tau} \int_{R_{n}} F^{2}(x, t) d x d t\right],
$$

and

$$
\chi(t)=C\left[K(t)+\tau^{2}-t^{2}+1\right] .
$$

From Gronwall's inequality it follows that

$$
E(\tau) \leqq f(\tau)+\int_{0}^{\tau} \chi(t) f(t) \exp \left(\int_{t}^{\tau} \chi(s) d s\right) d t
$$

and hence the uniform boundedness of $E(\tau)$ on $0 \leqq \tau \leqq T$.

In the proof of the theorem we will actually use the following immediate

CoROLLARY. Under the assumptions of the lemma we have

$$
\int_{0}^{T} \int_{R}\left[\varphi_{t}^{2}+\sum_{i=1}^{n} \varphi_{i}^{2}\right] d x d t \leqq \text { constant }
$$

The fact of crucial importance in this lemma, as far as the application to quasilinear equations is concerned, is that the bounds on the solution follow only from upper and not two-sided bounds on $\alpha_{t}^{i j}$.

We now come to the main result of this paper.

THEOREM. Weak solutions of (4) are uniquely determined by their initial conditions.

Proof. If $u^{1}(x, t)$ and $u^{2}(x, t)$ are two weak solutions of (4) with the same initial conditions we will show that if $\omega=u^{1}-u^{2}$, then

$$
\iint_{D} F(x, t) \omega(x, t) d x d t=0
$$

for every twice continuously differentiable function $F(x, t)$ having 
compact support in $D$ thus showing that $\omega$ is zero a.e. in $D$. For any test function $\varphi(x, t)$ we have

$$
\begin{aligned}
\iint_{D}\left\{\omega \varphi_{t t}\right. & +\sum_{i=1}^{n}\left[a^{i}\left(x, t, u^{1}, u_{k}^{1}\right)-a^{i}\left(x, t, u^{2}, u_{k}^{2}\right)\right] \varphi_{i} \\
- & {\left.\left[b\left(x, t, u^{1}\right)-b(x, t, u)\right] \varphi\right\} d x d t=0 . }
\end{aligned}
$$

Now

$$
\begin{aligned}
& a^{i}\left(x, t, u^{1}, u_{k}^{1}\right)-a^{i}\left(x, t, u^{2}, u_{k}^{2}\right) \\
& =\alpha^{i}(x, t) \omega+\sum_{j=1}^{n} \alpha^{i j}(x, t) \omega_{j}
\end{aligned}
$$

where

$$
\alpha^{i}(x, t)=\int_{0}^{1} \frac{\partial}{\partial u} \alpha^{i}\left(x, t, \tau u^{1}+(1-\tau) u^{2}, \tau u_{k}^{1}+(1-\tau) u_{k}^{2}\right) d \tau
$$

and

$$
\alpha^{i j}(x, t)=\int_{0}^{1} a^{i j}\left(x, t, \tau u^{1}+(1-\tau) u^{2}, \tau u_{k}^{1}+(1-\tau) u_{k}^{2}\right) d \tau .
$$

Similarly

$$
b\left(x, t, u^{1}\right)-b\left(x, t, u^{2}\right)=\beta(x, t) \omega
$$

where

$$
\beta(x, t)=\int_{0}^{1} \frac{\partial}{\partial u} b\left(x, t, \tau u^{1}+(1-\tau) u^{2}\right) d \tau .
$$

Thus for any test function $\varphi$ we have

$$
\iint_{D}\left\{\omega \varphi_{t t}+\sum_{i, j=1}^{n} \alpha^{i j} \omega_{j} \varphi_{i}+\sum_{i=1}^{n} \alpha^{i} \omega \varphi_{i}-\beta \omega \varphi\right\} d x d t=0 .
$$

The identity (17) will be established by constructing an appropriate sequence of test functions $\varphi^{m}(m=1,2, \cdots)$, using (22), and taking limits.

Let $\omega_{m}(x, t)$ be the Gaussian averaging kernel on $R_{n} \times(-\infty<$ $t<\infty)$ with support contained in the sphere

$$
|x|^{2}+t^{2} \leqq \frac{1}{m^{2}}
$$

thus

$$
\iint_{D} \omega_{m}(x, t) d x d t=1
$$


for all $m$. If the function $\psi(x, t)$ is in $L_{\mathrm{loc}}^{2}(\bar{D})$ we extend it to $R_{n} \times$ $(-\infty<t<\infty)$ by putting $\psi(x, t)=0$ for $t \leqq 0$ and then define

$$
\psi_{m}(x, t)=\psi * \omega_{m}
$$

where the $*$ indicates convolution. It is well known that the functions $\psi_{m}$ are smooth in $R_{n} \times(-\infty<t<\infty)$ and converge to $\psi$ in mean square on compact subsets of $\bar{D}$. If in addition the function $\psi$ is uniformly bounded on a compact subset of $\bar{D}$ then the functions $\psi_{m}$ possess the same bound on that subset.

The functions $\alpha_{m}^{i j}(x, t), \alpha_{m}^{i}(x, t)$ and $\beta_{m}(x, t)$ are now defined by the formulas for $\alpha^{i j}(x, t)$, etc., except that $u_{m}^{i}$ replaces $u^{i}(i=1,2)$. Using our assumptions it is easy to see that

$$
\begin{aligned}
& \left|\alpha_{m}^{i j}(x, t)-\alpha^{i j}(x, t)\right| \\
& \quad \leqq \operatorname{const}\left[\sum_{i=1}^{2}\left|u^{i}-u_{m}^{i}\right|+\sum_{i=1}^{2} \sum_{k=1}^{n}\left|u_{k}^{i}-u_{k, m}^{i}\right|\right],
\end{aligned}
$$

uniformly on compact subsets of $\bar{D}$, from which it follows that the sequence $\alpha_{m}^{i j}$ converges to $\alpha^{i j}$ in mean square on compact subsets of $\bar{D}$. Similarly, $\alpha_{m}^{i}$ and $\beta_{m}$ converge to $\alpha^{i}$ and $\beta$ in mean square on compact subsets of $\bar{D}$.

The test functions $\varphi^{m}$ are now chosen to satisfy the equation

$$
\varphi_{t t}^{m}-\sum_{i, j=1}^{n}\left(\alpha_{m}^{i j} \varphi_{i}^{m}\right)_{j}+\sum_{i=1}^{n} \alpha_{m}^{i} \varphi_{i}^{m}-\beta_{m} \varphi^{m}=F(x, t)
$$

and the conditions $\varphi^{m}(x, T)=\varphi_{t}^{m}(x, T)=0$, where it is assumed that the support of $F(x, t)$ is contained in $D \cap\{0<t<T\}$. Such functions are obtained by solving the backward initial-value problem with zero initial conditions at $t=T$. More precisely, we find solutions $\psi^{m}(x, t)$ of

$$
\psi_{t t}^{m}-\sum_{i, j=1}^{n}\left(\widetilde{\alpha}_{m}^{i j} \psi_{i}^{m}\right)_{j}+\sum_{i=1}^{n} \tilde{\alpha}_{m}^{i} \psi_{i}^{m}-\widetilde{\beta}_{m} \psi^{m}=F(x, t)
$$

on $0 \leqq t \leqq T$, subject to the initial conditions $\psi^{m}(x, 0)=\psi_{t}^{m}(x, 0)=0$, where $\widetilde{\alpha}_{m}^{i j}(x, t)=\alpha_{m}^{i j}(x, T-t)$, etc., and then put $\phi^{m}(x, t)=\psi^{m}(x, T-t)$. The standard existence theory [3] guarantees that we can find smooth solutions of this initial-value problem which are then admissible test functions.

From (22) and (24) we obtain, after some integration by parts, the identity

$$
\begin{aligned}
\iint_{D} \omega F d x d t= & \iint_{D}\left\{\sum_{i, j=1}^{n}\left(\alpha_{m}^{i j}-\alpha^{i j}\right) \omega_{j} \varphi_{i}^{m}\right. \\
& \left.+\sum_{i=1}^{n}\left(\alpha_{m}^{i}-\alpha^{i}\right) \omega \varphi_{i}^{m}+\left(\beta_{m}-\beta\right) \omega \varphi^{m}\right\} d x d t
\end{aligned}
$$


Our result will be established by showing that the right-hand side of (25) approaches zero as $m \rightarrow \infty$. Now as is easily seen,

$$
c_{1} \sum_{i=1}^{n} \xi_{i}^{2} \geqq \sum_{i, j=1}^{n} \widetilde{\alpha}_{m}^{i j}(x, t) \xi_{i} \xi_{j} \geqq c_{2} \sum_{i=1}^{n} \xi_{i}^{2}
$$

for all vectors $\left(\xi_{1}, \cdots, \xi_{n}\right)$, where $c_{1}$ and $c_{2}$ are constants independent of $m, F$ has compact support and the existence theorems then show that the supports of the functions $\psi^{m}$ are uniformly contained in some rectangular region $R \times[0, T]$ where $R$ is a fixed rectangle in $R_{n}$; the integration on the right-hand side of (25) need only be extended over this region. Since the functions $\omega$ and $\omega_{i}$ are uniformly bounded, and the functions $\alpha_{m}^{i j}$, etc., converge to $\alpha^{i j}$, etc., in mean square on $R \times[0, T]$ we see, using Schwarz' inequality, that it suffices to show that $\varphi_{i}^{m}$ and $\varphi^{m}$ are uniformly bounded in mean square over this region. This will be achieved by applying the Corollary of Lemma 1 to obtain a similar bound for the functions $\psi_{i}^{m}$ and $\psi^{m}$.

To apply Lemma 1 we need to show that assumptions $\mathrm{I}^{\prime}-\mathrm{VI}^{\prime}$ are satisfied by the coefficients of equation (23), with bounds independent of $m$. The only assumption that is not immediately evident is IV'. To establish it we note that

$$
\frac{\partial}{\partial t} \widetilde{\alpha}_{m}^{i j}(x, t)=-\frac{\partial}{\partial t} \alpha_{m}^{i j}(x, t)
$$

and so it suffices to demonstrate that

$$
\sum_{i, j=1}^{n} \frac{\partial}{\partial t} \alpha_{m}^{i j}(x, t) \xi_{i} \xi_{j} \geqq-K_{1}(t) \sum_{i=1}^{n} \xi_{i}^{2} .
$$

Now

$$
\begin{aligned}
\sum_{i, j=1}^{n} & \frac{\partial}{\partial t} \alpha_{m}^{i j}(x, t) \xi_{i} \xi_{j} \\
& =\sum_{i, j=1}^{n}\left[A^{i j}(x, t)+B^{i j}(x, t)+C^{i j}(x, t)\right] \xi_{i} \xi_{j}
\end{aligned}
$$

where

$$
\begin{aligned}
& A_{m}^{i j}(x, t) \\
& \quad=\int_{0}^{1} \frac{\partial a^{i j}}{\partial t}\left(x, t, \tau u_{m}^{1}+(1-\tau) u_{m}^{2}, \tau \frac{\partial u_{m}^{1}}{\partial x_{k}}+(1-\tau) \frac{\partial u_{m}^{2}}{\partial x_{k}}\right) \mathrm{d} \tau \\
& B_{m}^{i j}(x, t) \\
& \quad=\int_{0}^{1} \frac{\partial a^{i j}}{\partial u}(x, t, \cdots)\left[\tau \frac{\partial u_{m}^{1}}{\partial t}+(1-\tau) \frac{\partial u_{m}^{2}}{\partial t}\right] d \tau
\end{aligned}
$$


$C_{m}^{i j}(x, t)$
$=\sum_{k=1}^{n} \int_{0}^{1} \frac{\partial a^{i j}}{\partial u_{k}}\left[\tau \frac{\partial u_{m}^{1}}{\partial t \partial x_{k}}+(1-\tau) \frac{\partial u_{m}^{2}}{\partial t \partial x_{k}}\right] d \tau$

and we need only show the lower boundedness of the three separate quadratic forms. Since the $A^{i j}$ are uniformly bounded, the associated form is lower bounded. To show the lower boundedness of the two other forms we use assumptions IV and B. Using the properties of the averaging kernel it can be shown (see [6]) that the inequalities (9) imply that

$$
\frac{\partial u_{m}^{i}}{\partial t} \leqq \widetilde{K}(t)
$$

and

$$
\frac{\partial^{2} u_{m}^{i}}{\partial t \partial x_{k}} \leqq \widetilde{K}(t)
$$

where the function $\widetilde{K}(t) \in L^{1}(0, T)$. The same upper bound then holds for the convex combinations of these derivatives which occur in (26b) and (26c). Using IV we now see that

$$
\sum_{i, j=1}^{n}\left(B^{i j}+C^{i j}\right) \xi_{i} \xi_{j} \geqq-2 \widetilde{K}(t) \sum_{i=1}^{n} \xi_{i}^{2},
$$

completing the proof.

It is clear from the proof that the uniqueness theorem will still be valid if the inequalities in (7) are reversed and the inequalities (9) are replaced by lower bounds

$$
\begin{gathered}
\frac{u\left(x, t_{1}\right)-u\left(x, t_{2}\right)}{t_{1}-t_{2}} \geqq-K(t) \\
\frac{u_{k}\left(x, t_{1}\right)-u_{k}\left(x, t_{2}\right)}{t_{1}-t_{2}} \geqq-K(t) .
\end{gathered}
$$

Our assumptions were chosen to be consistent with those Oleǐnik [7]. In that paper she considered the system

$$
\begin{aligned}
\frac{\partial u}{\partial t}+\frac{\partial \varphi(x, t, v)}{\partial x} & =0 \\
\frac{\partial v}{\partial t}-\frac{\partial u}{\partial x} & =0 .
\end{aligned}
$$

For this system the proved uniqueness, under the assumption $\partial \varphi / \partial v<0$ and the convexity assumption $\partial^{2} \varphi / \partial v^{2}>0$, of pairs of weak solutions $(u, v)$ (defined in the obvious way), where $v$ satisfied a.e. a bound of 
the form

$$
\frac{v\left(x, t_{1}\right)-v\left(x, t_{2}\right)}{t_{1}-t_{2}}<K
$$

where $K$ is a constant. If we were dealing with smooth (i.e., twice continuously differentiable) solution pairs, then equations (27) could be replaced by the single equation

$$
\frac{\partial^{2} v}{\partial t^{2}}-\frac{\partial}{\partial x} a\left(x, t, v, v_{x}\right)=0
$$

where

$$
-a\left(x, t, v, v_{x}\right)=\frac{\partial}{\partial x} \varphi(x, t, v)=\frac{\partial \varphi}{\partial x}+\frac{\partial \varphi}{\partial v}(x, t, v) v_{x}
$$

Inequality (7a) is then

$$
\frac{\partial^{2} \varphi}{\partial v^{2}} \geqq 0
$$

which is even weaker than the strict convexity assumed by Oleinik, and inequality $(7 \mathrm{~b})$ is vacuously satisfied.

But this reduction can also be made for weak solutions. Using a result of Schauder [8] we see that the weak form of (27b) implies the existence of a (locally) Lipschitz continuous potential function $J(x, t)$ which a.e. satisfies $J_{x}=v$ and $J_{t}=u$. (This function can be normalized so that $J(0,0)=0$.) It is then easy to see that $J$ is a weak solution of

$$
J_{t t}+\frac{\partial}{\partial x} \varphi\left(x, t, J_{x}\right)=0
$$

with the initial conditions

$$
J(x, 0)=\int_{0}^{x} v(x, 0) d x
$$

and

$$
J_{t}(x, 0)=u(x, 0) \text {. }
$$

For (31a) the inequality (7b) is equivalent to (30) and Oleinnik's other assumptions are sufficient for the application of our theorem. The uniqueness theorem applied to (31) then generalizes Oleinik's result. I am indebted to E. D. Conway for pointing out the possibility of this reduction. 


\section{REFERENCES}

1. E. D. Conway, Generalized solutions of linear differential equations with discontinuous coefficients and the uniqueness question for multidimensional quasilinear conservation laws, J. Math. Anal. Appl. 18 (1967), 238-51.

2. E. D. Conway and J. Smoller, Global solution of the Cauchy problem for-quasilinear first order equations in several space variables, Comm. Pure Appl. Math. 19 (1966), 95-105.

3. R. Courant and D. Hilbert, Methods of Mathematical Physics, Vol. II, Interscience, New York, 1962.

4. J. Glimm, Solutions in the large for nonlinear hyperbolic systems of equations, Comm. Pure. Appl. Math. 18 (1965), 697-715.

5. A. E. Hurd, A uniqueness theorem for weak solutions of symmetric quasilinear hyperbolic systems, Pacific J. Math. 28 (1969), 555-559.

6. O. A. Oleinik, Discontinuous solutions of nonlinear differential equations, Uspehi Mat. Nauk (N.S.) 12 (1957), No. 3 (75), 3-73 (Amer. Math. Soc. Trans., series 2, Vol. 26, 95-172).

7. - On the uniqueness of the generalized solution of the Cauchy problem for a nonlinear system of equations occuring in mechanics, Uspehi Mat. Nauk (N.S.) 12 (1957), No. 6 (78), 169-176 (in Russian).

8. J. Schauder, Uber die Umkehrung eines Satzes aus der Variationsrechnung, Acta Szeged 3 (1928), 38-50.

9. J. Serrin, On the differentiability of functions of several variables, Arch. Rat. Mech. Anal. 7 (1961), 359-372.

Received September 13, 1968, and in revised form June 30, 1969. The preparation of this paper was sponsored in part by National Science Foundation Grant \$GP-5279.

University of CAlifornia, Los ANgeles 



\section{PACIFIC JOURNAL OF MATHEMATICS}

\section{EDITORS}

\author{
H. SAMELSON \\ Stanford University \\ Stanford, California 94305 \\ RICHARD PIERCE \\ University of Washington \\ Seattle, Washington 98105
}

J. DugundJI

Department of Mathematics University of Southern California Los Angeles, California 90007

BASIL GORDON*

University of California

Los Angeles, California 90024

\section{ASSOCIATE EDITORS}
E. F. BECKENBACH
B. H. NEUMANN
F. WOLE
K. YOSHIDA

\section{SUPPORTING INSTITUTIONS}

\author{
UNIVERSITY OF BRITISH COLUMBIA \\ CALIFORNIA INSTITUTE OF TECHNOLOGY \\ UNIVERSITY OF CALIFORNIA \\ MONTANA STATE UNIVERSITY \\ UNIVERSITY OF NEVADA \\ NEW MEXICO STATE UNIVERSITY \\ OREGON STATE UNIVERSITY \\ UNIVERSITY OF OREGON \\ OSAKA UNIVERSITY \\ UNIVERSITY OF SOUTHERN CALIFORNIA
}

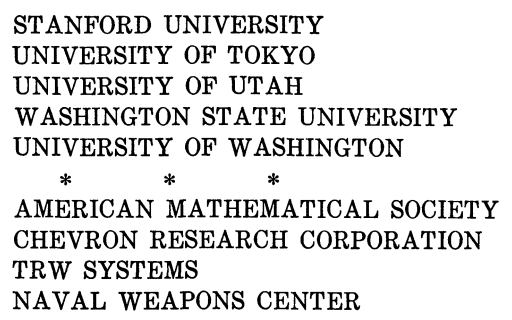

The Supporting Institutions listed above contribute to the cost of publication of this Journal, but they are not owners or publishers and have no responsibility for its content or policies.

Mathematical papers intended for publication in the Pacific Journal of Mathematics should be in typed form or offset-reproduced, (not dittoed), double spaced with large margins. Underline Greek letters in red, German in green, and script in blue. The first paragraph or two must be capable of being used separately as a synopsis of the entire paper. The editorial "we" must not be used in the synopsis, and items of the bibliography should not be cited there unless absolutely necessary, in which case they must be identified by author and Journal, rather than by item number. Manuscripts, in duplicate if possible, may be sent to any one of the four editors. Please classify according to the scheme of Math. Rev. 36, 1539-1546. All other communications to the editors should be addressed to the managing editor, Richard Arens, University of California, Los Angeles, California, 90024.

50 reprints are provided free for each article; additional copies may be obtained at cost in multiples of 50 .

The Pacific Journal of Mathematics is published monthly. Effective with Volume 16 the price per volume (3 numbers) is $\$ 8.00$; single issues, $\$ 3.00$. Special price for current issues to individual faculty members of supporting institutions and to individual members of the American Mathematical Society: $\$ 4.00$ per volume; single issues $\$ 1.50$. Back numbers are available.

Subscriptions, orders for back numbers, and changes of address should be sent to Pacific Journal of Mathematics, 103 Highland Boulevard, Berkeley, California, 94708.

PUBLISHED BY PACIFIC JOURNAL OF MATHEMATICS, A NON-PROFIT CORPORATION

Printed at Kokusai Bunken Insatsusha (International Academic Printing Co., Ltd.), 7-17, Fujimi 2-chome, Chiyoda-ku, Tokyo, Japan.

* Acting Managing Editor. 


\section{Pacific Journal of Mathematics}

\section{Vol. 32, No. $2 \quad$ February, 1970}

Harry P. Allen and Joseph Cooley Ferrar, Jordan algebras and exceptional subalgebras of the exceptional algebra $E_{6} \ldots \ldots \ldots \ldots \ldots \ldots \ldots 283$

David Wilmot Barnette and Branko Grünbaum, Preassigning the shape of a

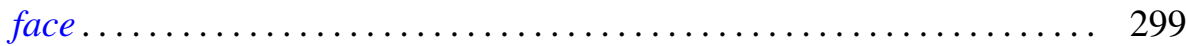

Robert Francis Craggs, Involutions of the 3-sphere which fix 2-spheres . . . . 307

David William Dean, Bor-Luh Lin and Ivan Singer, On k-shrinking and $k$-boundedly complete bases in Banach spaces ................ 323

Martin Engert, Finite dimensional translation invariant subspaces ....... 333

Kenneth Lewis Fields, On the global dimension of residue rings ......... 345

Howard Gorman, The Brandt condition and invertibility of modules ....... 351

Benjamin Rigler Halpern, A characterization of the circle and interval ..... 373

Albert Emerson Hurd, A uniqueness theorem for second order quasilinear

hyperbolic equations ............................... 415

James Frederick Hurley, Composition series in Chevalley algebras ...... 429

Meira Lavie, Disconjugacy of linear differential equations in the complex

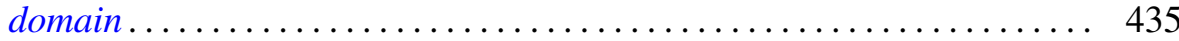

Jimmie Don Lawson, Lattices with no interval homomorphisms ......... 459

Roger McCann, A classification of center-foci ................. 467

Evelyn Rupard McMillan, On continuity conditions for functions . . . . . . . 479

Graciano de Oliveira, A conjecture and some problems on permanents .... 495

David L. Parrott and S. K. Wong, On the Higman-Sims simple group of order $44,352,000$.

Jerome L. Paul, Extending homeomorphisms ................. 517

Thomas Benny Rushing, Unknotting unions of cells .............. 521

Peter Russell, Forms of the affine line and its additive group.......... 527

Niel Shilkret, Non-Archimedean Gelfand theory ................. 541

Alfred Esperanza Tong, Diagonal submatrices of matrix maps.......... 551 